prevalence or severity of AMS, it did increase the prevalence of nongastrointestinal symptoms such as headache, fatigue, and dizziness (score $3.3 \pm 1.7$ vs $4.3 \pm 1.6$ exercise, $P \quad .04$ ).

The authors concluded that cardiac autonomic modulation during hypoxia does not influence the development of AMS. They speculated that the sympathetic predominance of moderate exercise may have a protective effect against nausea and vomiting while worsening nongastrointestinal symptoms of AMS.

(Int J Sports Med. 2013 Feb 5 [Epub ahead of print]) K Mairer, M Wille, W Grander, M Burtscher.

Prepared by Adam Blanchard, MD, University of Utah Emergency Medicine Resident, Salt Lake City, UT, USA.

\section{JOURNAL OF TRAUMA AND ACUTE CARE SURGERY} Safety and Efficacy of Oral Transmucosal Fentanyl Citrate
for Prehospital Pain Control on the Battlefield

Morphine has been the mainstay for prehospital battlefield analgesia, with its use dating back to the War of 1812 and the American Civil War. Although morphine is an excellent analgesic, its significant adverse effects include cardiorespira tory depression, immune function suppression, and titration difficulties. Oral transmucosal fentanyl citrate (OTFC) is a potent alternative with a faster onset and fewer adverse effects.

This study was a retrospective analysis of the prehospital use of OTFC in Afghanistan and Iraq from 2003 to 2010. In all, 286 patients received OTFC for various injuries, with traumatic extremity injuries being the most common. The primary outcome was pain assessment using the numerical rating system at 0,15 , and 30 minutes after OTFC administration. Continuous pulse oximetry was utilized, and adverse events were recorded. The OTFC lowered pain scores in $97 \%$ of patients, with a mean dose of $962.4 \mu \mathrm{g}$. Approximately $18 \%$ of patients required other analgesics for adequate pain control. Only 1 significant adverse event was recorded in the setting of hypoventilation, requiring low dose naloxone. Minor adverse events included nausea, pruritus, drowsiness, and dizziness. Major benefits of OTFC included ease of administration, rapid onset, and ability to titrate safely and effectively.

Limitations of the study include retrospective design, inadequate documentation, and limited follow up data. The authors recognized the potential use OTFC for acute pain in civilian prehospital and austere environments.

(J Trauma Acute Care Surg. 2012;73[suppl 5]:490 495) IS Wedmore, RS Kotwal, JG McManus, et al. Prepared by Joseph Krakker, Rocky Vista University College of Osteopathic Medicine Medical Student, Parker, CO, USA.

\section{RESUSCITATION}

\section{Resuscitation of Avalanche Victims: Evidence-based Guidelines of the International Commission for Mountain Emergency Medicine (ICAR MEDCOM), Intended for Physicians and Other Advanced Life Support Personnel}

Avalanches are becoming more common and kill approxi mately 150 people in North America and Europe each year. There are several guidelines published regarding on site management and transport of avalanche victims, most recently in 2010 from the International Liaison Committee on Resus citation. The authors' goal was to develop evidence based guidelines for $27 \mathrm{key}$ aspects of avalanche rescue and to obtain a consensus for the International Commission for Mountain Emergency Medicine. After an extensive literature search, 96 high quality and relevant articles were chosen for full review.

Excluding lethal trauma and clearly nonsurvivable condi tions, the investigators found that avalanche victim survival rate is $77 \%$ and is primarily determined by the grade of burial, duration of burial, asphyxia, core temperature, and trauma. Interestingly, hypothermia is rarely the primary cause of death if the victim's airway is patent. Grade of burial was the strongest predictor of survival, with $47.6 \%$ survival rate for complete burials vs $95.8 \%$ for partial burials. Prior studies have shown the survival rates precipitously drop after approximately 10 to 18 minutes of burial. The authors proposed a 35 minute burial time to guide rescue efforts: for victims buried less than 35 minutes found in cardiac arrest, asphyxia should be assumed and cardiopulmonary resuscitation should be started. Advanced airway intervention should be considered if the rescuer is competent; however, inexperienced rescuers should continue mouth to mouth or bag mask ventilation. Guidelines for termination of cardio pulmonary resuscitation include: lethal injury; completely frozen, blocked airway; burial time more than 35 minutes; serum potassium greater than $12 \mathrm{mmol} / \mathrm{L}$; or unacceptable risk to the rescuing team. After adhering to appropriate spinal precautions and insulating against further heat loss, patients who can potentially survive should be carefully transported in a horizontal position to a nearby hospital with proper capabilities. This is a partial list of the primary recommenda tions; please review the article in detail before changing practice patterns.

(Resuscitation. 2012 Nov 2 [Epub ahead of print]) H Brugger, B Durrer, F Elsensohn, et al.

Prepared by Matthew Stewart, MD, University of Utah Emergency Medicine Resident, Salt Lake City, UT, USA. 